\title{
SETS OF UNIQUENESS IN COMPACT, 0-DIMENSIONAL METRIC GROUPS
}

\author{
D. J. GRUBB
}

\begin{abstract}
An investigation is made of sets of uniqueness in a compact 0dimensional space. Such sets are defined by pointwise convergence of sequences of functions that generalize partial sums of trigonometric series on Vilenkin groups. Several analogs of classical uniqueness theorems are proved, including a version of N. Bary's theorem on countable unions of closed sets of uniqueness.
\end{abstract}

Introduction. The theory of sets of uniqueness in the case of classical trigonometric series has a long history and many beautiful results. In recent years, some of these results have been carried over to the context of Walsh series. The goal of this paper is to generalize the Walsh series results to the case of infinite, compact, 0-dimensional metric groups. The methods will be quite different from those usually used in the study of Walsh series. Where before, problems were transferred to the unit interval for consideration, now everything can be done on the group itself. This simplifies many of the proofs and provides a substantial change in the philosophy of approach to uniqueness problems.

Our main concept is that of a quasi-measure, Any regular, Borel measure may be regarded as a quasi-measure. but not conversely. We will characterize positive measures among the quasi-measures and use this characterization to obtain our uniqueness results.

One of the remarkable facts about this theory is that no explicit group structure is needed in its development. The first section is therefore devoted to defining the basic concepts, such as quasi-measures and partial sums of trigonometric series, in the context of infinite, compact, 0 -dimensional, metric spaces with a certain type of martingale structure.

ACKNOWLEDGMENTS. I would like to thank Karl Stromberg for his guidance and Ben Lange for many fruitful discussions.

1. Basic definitions. Let $X$ be a compact, 0-dimensional, metric space. Suppose that a sequence of covers of $X,\left\{C_{n}\right\}_{n=0}^{\infty}$, is given such that

(a) Elements of a given $C_{n}$ are disjoint and clopen,

(b) Each element of $C_{n}$ is properly contained in some (unique) element of $C_{n-1}$ for $n \geq 1$,

(c) $C_{0}=\{X\}$

(d) $\bigcup_{n=0}^{\infty} C_{n}$ is a base for the topology of $X$.

For $x \in X$ and $n \geq 0$, denote by $U(n, x)$ that element of $C_{n}$ which contains $x$. Since each $C_{n}$ is a cover by disjoint sets, $U(n, x)$ is well defined.

Received by the editors May 5, 1986.

1980 Mathematics Subject Classification (1985 Revision). Primary 43A46.

Key words and phrases. Vilenkin group, set of uniqueness. 
We also assume that a Borel probability measure, $\lambda$, is given on $X$ such that

(e) For $n \geq 0$ and $U, V \in C_{n}$, we have $\lambda(U)=\lambda(V)>0$.

Thus, all elements of $C_{n}$ are given the same mass by $\lambda$. We let $a_{n}$ denote this common mass, so $U \in C_{n}$ implies $\lambda(U)=a_{n}$.

From now on, we assume that $X$, the sequence $\left\{C_{n}\right\}_{n=0}^{\infty}$, and the measure $\lambda$ are fixed.

Notation. We let $\xi_{A}$ denote the characteristic (indicator) function for a subset $A$ of $X$.

DEFINITION 1.1. Let $\mathrm{TP}(X)$ be the linear span of $\xi_{U}$ where $U$ ranges over $\bigcup_{n=0}^{\infty} C_{n}$. Since each such $U$ is clopen, $\operatorname{TP}(X)$ is a linear subspace of $C(X)$, the continuous, complex-valued functions on $X$. Since $\bigcup_{n=0}^{\infty} C_{n}$ generates the topology of $X$, this subspace is dense in the uniform norm on $C(X)$. We call elements of $\mathrm{TP}(X)$ trigonometric polynomials. The reason for this terminology will become apparent later.

DEFINITION 1.2. Let $\mathrm{QM}(X)=\mathrm{TP}(X)^{*}$ be the linear space dual of $\mathrm{TP}(X)$. Call elements of $\mathrm{QM}(X)$ quasi-measures. If $S$ is a quasi-measure and $f$ is a trigonometric polynomial, we let $(S, f)$ denote the value of $S$ at $f$. One way of defining a quasi-measure is to specify $\left(S, \xi_{U}\right)$ for $U \in \bigcup_{n=0}^{\infty} C_{n}$. The matching condition

$$
\left(S, \xi_{U}\right)=\sum\left\{\left(S, \xi_{V}\right): V \in C_{n+1}, V \subseteq U\right\}
$$

for $U \in C_{n}$ must be checked for well-definedness of the quasi-measure.

If $S$ is a quasi-measure, we define the real and imaginary parts of $S$ by

$$
\left(\operatorname{Re} S, \xi_{U}\right)=\operatorname{Re}\left(S, \xi_{U}\right)
$$

and

$$
\left(\operatorname{Im} S, \xi_{U}\right)=\operatorname{Im}\left(S, \xi_{U}\right)
$$

for $U \in \bigcup_{n=0}^{\infty} C_{n}$. Then $\operatorname{Re} S$ and $\operatorname{Im} S$ are both quasi-measures and $S=\operatorname{Re} S+$ $i \operatorname{Im} S$.

Definition 1.3. If $S$ is a quasi-measure, $x$ an element of $X$, and $n \geq 0$, we define the $n$th partial sum of the Fourier series of $S$ at $x$ to be

$$
s_{n}(S, x)=s_{n}(S)(x)=\left(S, \xi_{U(n, x)}\right) / a_{n} .
$$

Recall that $U(n, x)$ is the element of $C_{n}$ which contains $x$ and $a_{n}=\lambda(U(n, x))$. Notice that $s_{n}(S)$ is constant on elements of $C_{n}$, which shows that $s_{n}(S)$ is a trigonometric polynomial for all $n \geq 0$. Also, notice that $s_{n}(\operatorname{Re} S)$ and $s_{n}(\operatorname{Im} S)$ are real valued.

Finally, we give several examples which serve to motivate our terminology and to provide the main justification for this paper.

EXAMPLES. Let $G$ be an infinite, compact, 0-dimensional, metric group, not necessarily abelian. Using the metric and connectivity properties, it is possible to find a sequence of clopen, normal subgroups $\left\{H_{n}\right\}_{n=0}^{\infty}$ of $G$ such that

( $\left.\mathrm{a}^{\prime}\right) H_{0}=G$,

(b') $H_{n+1} \subsetneq H_{n}$ for $n \geq 0$, and

$\left(\mathrm{c}^{\prime}\right) \bigcap_{n=0}^{\infty} H_{n}=\{e\}$, where $e$ is the identity of $G$.

If we let $C_{n}=\left\{x H_{n}: x \in G\right\}$, we obtain a sequence of covers $\left\{C_{n}\right\}_{n=0}^{\infty}$ satisfying conditions (a) $-(\mathrm{d})$. In this case $U(n, x)=x H_{n}$. 
If we let $\lambda$ be Haar measure on $G$, then condition (e) above is also satisfied.

(2) If the group of the previous example is abelian (in other words, if $G$ is a Vilenkin group), we let $\Gamma$ be its Pontryagin dual. Then $\Gamma$ is countable and discrete. If we let $H_{n}^{\perp}$ denote the annihilator of $H_{n}$ in $\Gamma$, i.e.,

$$
H_{n}^{\perp}=\left\{\gamma \in \Gamma: \gamma=1 \text { on } H_{n}\right\} \text {. }
$$

then,

(a") $H_{0}^{\perp}=\{1\}$, where 1 is the constant character,

(b") $H_{n}^{\perp} \subsetneq H_{n+1}^{\perp}$ for $n \geq 0$, and

(c") $\bigcup_{n=0}^{\infty} H_{n}^{\perp}=\Gamma$.

In this setting, there is already a definition of trigonometric polynomials as finite linear combinations of characters. To see that our definition is equivalent to the standard one, the following identities are used:

$$
\begin{gathered}
\xi_{x H_{n}}=\lambda\left(H_{n}\right) \sum_{\gamma \in H_{n}^{\perp}} \overline{\gamma(x)} \gamma, \\
\gamma=\sum\left\{\gamma(x) \xi_{x H_{n}}: x H_{n} \in C_{n}\right\} \quad \text { if } \gamma \in H_{n}^{\perp} .
\end{gathered}
$$

To understand the concept of a quasi-measure in this setting, let $q: \Gamma \rightarrow \mathbf{C}$ be any complex-valued function on $\Gamma$. We may define $m_{q} \in \mathrm{QM}(G)$ by setting

$$
\begin{aligned}
\left(m_{q}, \xi_{x H_{n}}\right) & =\lim _{k \rightarrow \infty} \int_{x H_{n}}\left(\sum_{\gamma \in H_{k}^{\perp}} q(\gamma) \gamma\right) d \lambda \\
& =\sum_{\gamma \in H_{n}^{\perp}} q(\gamma) \gamma(x) \lambda\left(H_{n}\right) .
\end{aligned}
$$

The second equality holds since

$$
\int_{x H_{n}} \gamma d \lambda= \begin{cases}\gamma(x) \lambda\left(H_{n}\right) & \text { if } \gamma \in H_{n}^{\perp}, \\ 0 & \text { if } \gamma \notin H_{n}^{\perp} .\end{cases}
$$

This shows that the limit above exists. The matching condition (1.1) is satisfied because of the additivity properties of the integral. If we call $q$ the Fourier transform of $m_{q}$, then

$$
s_{n}\left(m_{q}, x\right)=\sum_{\gamma \in H_{n}^{\perp}} q(\gamma) \gamma(x)
$$

is actually a partial sum of the Fourier series of $m_{q}$. This provides the motivation for our terminology in the general case.

(3) A particular case of the previous example which has been studied is $G=$ $\prod_{k=0}^{\infty} \mathbf{Z} /(p)$. If we choose $H_{n}=\prod_{k=n}^{\infty} \mathbf{Z} /(p)$, properties $\left(\mathrm{a}^{\prime}\right)-\left(\mathrm{c}^{\prime}\right)$ are fulfilled. In this case, quasi-measures were defined and studied in $[\mathbf{1}]$. For the case $p=2$, the results may be understood in terms of Walsh series. Quasi-measures in this case were studied in $[\mathbf{2}]$.

2. Convergence properties. Let $\mu \in M(X)$, the set of Borel measures on $X$. We may regard $\mu$ as a quasi-measure via

$$
(\mu, f)=\int_{X} f d \mu \quad \text { for } f \in \operatorname{TP}(X) .
$$


Then

$$
s_{n}(\mu, x)=\left(\mu, \xi_{U(n, x)}\right) / a_{n}=\mu(U(n, x)) / \lambda(U(n, x)) .
$$

Note that if $\mu, \nu \in M(X)$ and $s_{n}(\mu, x)=s_{n}(\nu, x)$ for all $x \in X$ and $n \geq 0$, then $\mu=\nu$, since then $\mu(U(n, x))=\nu(U(n, x))$ for all $x$ in $X$ and $n \geq 0$.

We may regard elements of $L^{1}(\lambda)$ as measures, and thus as quasi-measures via

$$
(f, g)=(f d \lambda, g)=\int_{X} f g d \lambda \quad \text { for } g \in \operatorname{TP}(X) .
$$

In the case of the Fourier series of a continuous function, the following result is obtained. See [3] for the Walsh series case.

THEOREM 2.1. If $f \in C(X)$, then $s_{n}(f)$ converges to $f$ uniformly.

PROOF.

$$
\begin{aligned}
\left|f(x)-s_{n}(f, x)\right| & =\left|f(x)-\frac{1}{a_{n}} \int_{U(n, x)} f d \lambda\right| \\
& =\frac{1}{a_{n}}\left|\int_{U(n, x)}(f(x)-f) d \lambda\right| \leq \frac{1}{a_{n}} \int_{U(n, x)}|f(x)-f| d \lambda \\
& \leq \sup \{|f(x)-f(y)|: y \in U(n, x)\}
\end{aligned}
$$

Since $X$ is compact and metric, $f$ is uniformly continuous. The last term therefore goes to 0 as $n$ goes to $\infty$. This convergence is uniform in $x$.

While this is a very easy result, it underlies some fundamental differences between the classical Fourier series and the present field of investigation. In the classical theory, even pointwise convergence of a Fourier series cannot be guaranteed, let alone uniform convergence. Essentially, the present case has a "Dirichlet kernel" that acts like a "Fejér kernel."

Our next result requires a standard martingale convergence theorem for its proof. The result needed is Theorem 20.56 on p. 369 of [4].

THEOREM 2.2. Let $\mu \in M(X)$. Write $\mu=\mu_{s}+f d \lambda$ where $\mu_{s} \perp \lambda$ and $f \in$ $L^{1}(\lambda)$. Then

(a) $\lim _{n \rightarrow \infty} s_{n}(\mu, x)=f(x) \lambda$-a.e.

(b) $\lim _{n \rightarrow \infty}\left|s_{n}(\mu, x)\right|=+\infty \mu_{s}$-a.e.

In particular, the Fourier series of a $\lambda$-integrable function converges $\lambda$-a.e. to that function.

ProOF. Each cover $C_{n}$ generates a finite $\sigma$-algebra of subsets of $X$, call this $\sigma$-algebra $M_{n}$. Then $M_{n} \subsetneq M_{n+1}$ and $B(X)$, the Borel sets on $X$, is the smallest $\sigma$-algebra containing every $M_{n}$.

If $\mu_{n}$ is the restriction of $\mu$ to $M_{n}$, and $\lambda_{n}$ is a similar restriction of $\lambda$, then the Radon-Nikodým derivative of $\mu_{n}$ with respect to $\lambda_{n}$ is simply $s_{n}(\mu)$ by formula (2.2). The result follows from the martingale result.

NOTE. This theorem is proven in a different way (and in a much more general context) in $\S 44$ of [5]. 


\section{Characterizing positive measures among quasi-measures.}

THEOREM 3.1. Let $S \in \mathrm{QM}(X)$ and assume that there is a real number $M$ such that

$$
\int_{X}\left|s_{N}(S)\right| d \lambda \leq M \quad \text { for all } n \geq 0 .
$$

Then $S \in M(X)$. See Theorem 6 of [6] for the Walsh series case.

Proof. Define $\mu_{n} \in M(X)$ by $\mu_{n}=s_{n}(S) \lambda$. Then $\left\|\mu_{n}\right\| \leq M$ for all $n \geq 0$. By Alaoglu-Bourbaki, there is a weak* cluster point, $\mu$, of $\left\{\mu_{n}\right\}_{n=0}^{\infty}$ in $M(X)=C(X)^{*}$, the Banach space dual of $C(X)$.

Then for $U \in C_{k}, x \in U$, and $n \geq k$,

$$
\begin{aligned}
\left(S, \xi_{U}\right) & =\sum_{\{}\left\{\left(S, \xi_{V}\right): V \in C_{n}, V \subseteq U\right\}=\sum_{X}\left\{a_{n} s_{n}(S, y): U(n, y) \subseteq U\right\} \\
& =\int_{U} s_{n}(S) d \lambda=\int_{X} \xi_{U} s_{n}(S) d \lambda \rightarrow \int_{X} \xi_{U} d \mu=\left(\mu, \xi_{U}\right),
\end{aligned}
$$

where the convergence is of an appropriate subsequence. Thus $S=\mu$ as quasimeasures.

Next, we find a simple characterization of the positive measures in terms of their Fourier series.

THEOREM 3.2. Let $S \in \mathrm{QM}(X)$ be such that each $s_{n}(S)$ is real valued and assume

$$
\varlimsup_{n \rightarrow \infty} s_{n}(S, x) \geq 0 \quad \text { for each } x \in X .
$$

Then $S \in M^{+}(X)$, i.e. $S$ is a positive measure.

PROOF. First, notice that for $\alpha>0, s_{n}(\alpha \lambda)=\alpha$ for all $n \geq 0$. Thus

$$
\varlimsup_{n \rightarrow \infty} s_{n}(S+\alpha \lambda, x) \geq \alpha>0 \quad \text { for all } x \in X .
$$

If we show that $S+\alpha \lambda \in M^{+}(X)$, we may let $\alpha$ go to 0 to get $S \in M^{+}(X)$. We may therefore assume that strict inequality holds in formula (3.2).

Under this assumption, fix $x \in X$ and $n \geq 0$. For each $y \in U(n, x)$, we may find $k_{y} \geq n$ such that $s_{k_{y}}(S, y)>0$.

Then, since $U(n, x)$ is compact and $\left\{U\left(k_{y}, y\right)\right\}_{y \in U(n, x)}$ covers $U(n, x)$, we may find a finite subcover. Since two elements of $\bigcup_{n=0}^{\infty} C_{n}$ are either disjoint or one contains the other, this cover may be chosen with disjoint elements $\left\{U\left(k_{j}, y_{j}\right)\right\}_{j=1}^{p}$. Then

$$
a_{n} s_{n}(S, x)=\left(S, \xi_{U(n, x)}\right)=\sum_{j=1}^{p}\left(S, \xi_{U\left(k_{j}, y_{j}\right)}\right)=\sum_{j=1}^{p} a_{k_{j}} s_{k_{j}}\left(S, y_{j}\right)>0 .
$$

Thus $s_{n}(S, x)>0$ for every $n \geq 0$ and $x \in X$.

Now,

$$
\begin{aligned}
\int_{X}\left|s_{n}(S, x)\right| d \lambda(x) & =\int_{X} s_{n}(S, x) d \lambda(x)=\sum\left\{a_{n} s_{n}(S, y): U(n, y) \in C_{n}\right\} \\
& =\sum\left\{\left(S, \xi_{U(n, y)}\right): U(n, y) \in C_{n}\right\}=\left(S, \xi_{X}\right) \quad \text { for } n \geq 0 .
\end{aligned}
$$


By Theorem 3.1, we have $S \in M(X)$. For $U \in C_{n}, S(U)=\left(S, \xi_{U}\right)=$ $a_{n} s_{n}(S, x)>0$ for $x \in U$. Thus $S$ is a positive measure.

EXAMPLE. The limit hypothesis above cannot be relaxed at even one point of $X$ without some additional hypothesis being added. To see this, pick $x_{0} \in X$, and let $\mu=-\delta_{x_{0}}$, where $\delta_{x_{0}}$ is the unit point mass at $x_{0}$. Then

$$
s_{n}(\mu, x)= \begin{cases}-1 / a_{n} & \text { if } x_{0} \in U(n, x), \\ 0 & \text { if } x_{0} \notin U(n, x) .\end{cases}
$$

Then $\lim _{n \rightarrow \infty} s_{n}(\mu, x)=0$ for $x \neq x_{0}$, yet $\mu \notin M^{+}(X)$. The problem here is that we allow discrete measures as candidates in our discussion. To remedy this situation, we first give a lemma to increase understanding of the nature of discrete measures.

LEMMA 3.3. Let $\mu \in M(X)$. Then

$$
\lim _{n \rightarrow \infty} a_{n} s_{n}(\mu, x)=\mu(\{x\}) .
$$

In particular, continuous measures are characterized by the condition

$$
\lim _{n \rightarrow \infty} a_{n} s_{n}(\mu, x)=0 \quad \text { for all } x \in X .
$$

PROOF. Since $\bigcap_{n=0}^{\infty} U(n, x)=\{x\}, \lim _{n \rightarrow \infty} a_{n} s_{n}(\mu, x)=\lim _{n \rightarrow \infty} \mu(U(n, x))$ $=\mu(\{x\})$.

Now we have

THEOREM 3.4. Let $S \in \mathrm{QM}(X)$ be such that each $s_{n}(S)$ is real valued. Suppose that there is a countable set $C$ of $X$ such that

(a) $\varlimsup_{n \rightarrow \infty} s_{n}(S, x) \geq 0$ for $x \notin C$, and

(b) $\lim _{n \rightarrow \infty} a_{n} s_{n}(S, x)=0$ for $x \in C$.

Then $S \in M^{+}(X)$.

ProOF. Let $\mu$ be any measure concentrated on $C$ with $\mu(\{x\})>0$ for $x$ in $C$. Then, for $\alpha>0, s_{n}(\alpha \mu)$ is real valued and $\overline{\lim }_{n \rightarrow \infty} s_{n}(\alpha \mu, x) \geq 0$ for $x \in X$. Thus $s_{n}(S+\alpha \mu)$ is real valued and $\varlimsup_{n \rightarrow \infty} s_{n}(S+\alpha \mu, x) \geq 0$ for $x \notin C$.

But

$$
\begin{aligned}
\lim _{n \rightarrow \infty} a_{n} s_{n}(S+\alpha \mu, x) & =\lim _{n \rightarrow \infty} a_{n} s_{n}(S, x)+\lim _{n \rightarrow \infty} a_{n} s_{n}(\alpha \mu, x) \\
& =\alpha \mu(\{x\})>0 \text { for } x \in C .
\end{aligned}
$$

This shows that $\lim _{n \rightarrow \infty} s_{n}(S+\alpha \mu, x)>0$ for $x \in C$ also. By Theorem 3.2, $S+\alpha \mu \in M^{+}(X)$. Letting $\alpha$ go to 0 gives the result.

Two corollaries of the last theorems are of independent interest. They are both essentially uniqueness results.

COROLlary 3.5. Let $S \in \mathrm{QM}(X)$ be such that $\lim _{n \rightarrow \infty} s_{n}(S, x)=0$ for all $x \in X$. Then $S=0$.

ProOF. Use Theorem 3.2 on the real and imaginary components of both $S$ and $-S$. 
COROLlaRY 3.6. Let $S \in \mathrm{QM}(X)$ be such that $\lim _{n \rightarrow \infty} a_{n} s_{n}(S, x)=0$ for all $x \in X$. If $\lim _{n \rightarrow \infty} s_{n}(S, x)=0$ except for countably many $x \in X$, then $S=0$.

4. Uniqueness. In this section, we will derive several analogs of classical uniqueness theorems (see Chapter 9 of [7]) as well as generalizations of several results on Walsh series. Our main results are Theorem 4.2 , which is essentially a theorem on the uniqueness of the expansion of an integrable function in terms of a trigonometric series, and Theorem 4.6, which is an analog of N. Bary's theorem that a countable union of closed $U$-sets on the circle group is again a $U$-set. Previously, these results were obtained by transferring consideration to the unit interval. Then a detailed analysis of dyadic derivatives of indefinite integrals provided the theorems. In this section, we circumvent this procedure by remaining on the original space but using the results from the last section.

To start this program, we need a lemma.

LEMMA 4.1. If $f$ is a $\lambda$-integrable function which is upper semicontinuous on $X$, then

$$
\varlimsup_{n \rightarrow \infty} s_{n}(f, x) \leq f(x) \quad \text { for all } x \in X .
$$

ProOF. Assume $f$ is upper semicontinuous, $x_{0} \in X$, and $f\left(x_{0}\right)<\alpha$. Find $n_{0}$ such that $x \in U\left(n_{0}, x_{0}\right)$ implies $f(x)<\alpha$. Then for $n \geq n_{0}$,

$$
s_{n}\left(f, x_{0}\right)=\frac{1}{a_{n}} \int_{U\left(n, x_{0}\right)} f d \lambda<\alpha .
$$

Thus $\varlimsup_{n \rightarrow \infty} s_{n}\left(f, x_{0}\right) \leq \alpha$. Taking the infimum over $\alpha>f\left(x_{0}\right)$ gives the result.

The next result generalizes Theorem 3 of $[\mathbf{8}]$ (see also [9]), which covers the Walsh series case.

THEOREM 4.2. Let $S \in \mathrm{QM}(X)$ be such that $s_{n}(S)$ is real valued for every $n \geq 0, g: X \rightarrow \mathbf{R}$ be in $L^{1}(\lambda)$, and $C \subseteq X$ be countable. If

(a) $\lim _{n \rightarrow \infty} a_{n} s_{n}(S, x)=0$ for $x \in C$,

(b) $\varlimsup_{n \rightarrow \infty}\left|s_{n}(S, x)\right|<\infty$ for $x \notin C$, and

(c) $g(x) \leq \varlimsup_{n \rightarrow \infty} s_{n}(S, x)$ for $x \notin C$, then $S \in L^{\overline{1}}(\lambda)$. Then

ProOF. Let $f \leq g$ be a $\lambda$-integrable function which is upper semicontinuous.

$\varlimsup_{n \rightarrow \infty} s_{n}(S-f, x) \geq \varlimsup_{n \rightarrow \infty} s_{n}(S, x)-\varlimsup_{n \rightarrow \infty} s_{n}(f, x) \geq g(x)-f(x) \geq 0 \quad$ for $x \in C$.

Also,

$$
\lim _{n \rightarrow \infty} a_{n} s_{n}(S-f, x)=\lim _{n \rightarrow \infty} a_{n} s_{n}(S, x)-\lim _{n \rightarrow \infty} a_{n} s_{n}(f, x)=0 \quad \text { for } x \in C .
$$

By Theorem 3.4, $S-f \in M^{+}(X)$. Thus $S \in M(X)$. Write $S=S_{a}+S_{s}$ where $S_{a}<<\lambda$ and $S_{s} \perp \lambda$. Then, by Theorem 2.2 ,

$$
\lim _{n \rightarrow \infty}\left|s_{n}(S, x)\right|=+\infty \quad S_{s} \text {-a.e. }
$$

By assumption (b), $S_{s}$ is concentrated on $C$, so $S_{s}$ is discrete. But now, Lemma 3.3 and assumption (a) show $S_{s}=0$. Thus $S=S_{a} \in L^{1}(\lambda)$. 
This theorem is an analog of a classical result, but the only case where it was known for totally disconnected groups was the Walsh series case. Even there the proofs were long and tedious because of the transfer to the unit interval.

The next result is also an analog of a classical result. For the case $G=$ $\prod_{n=0}^{\infty} \mathbf{Z} /(p)$, see [1], from which the proof is adapted.

THEOREM 4.3. Let $S \in \mathrm{QM}(X), U \in C_{N_{0}}$, and assume $\lim _{n \rightarrow \infty} s_{n}(S, x)=$ $0 \lambda$-a.e. on $U$. If, furthermore, $\varlimsup_{n \rightarrow \infty}\left|s_{n}(S, x)\right|<\infty$ for all $x \in U$, then $\left(S, \xi_{V}\right)=$ 0 for all $V \subseteq U, V \in C_{m}, m \geq N_{0}$. In particular, if $U=X \in C_{0}, S=0$.

Proof. It is enough to show that $\left(S, \xi_{U}\right)=0$ since any $V$ as above satisfies the same hypotheses as $U$. Set $U_{0}=U$ and $b_{n}=a_{n}^{-1}$.

Let $0<\varepsilon<1$. Set $\varepsilon_{1}=\varepsilon / 2 a_{N_{0}}$. By Egorov's Theorem, there is a compact subset $E$ of $U$ such that $\lambda(E)>(1-\varepsilon) a_{N_{0}}$ and $s_{n}(S, x) \rightarrow 0$ uniformly on $E$. Thus, there is an $N_{1}>N_{0}$ such that $\left|s_{N_{1}}(S, x)\right|<\varepsilon_{1}$ for $x \in E$.

Set $Z_{1}=\left\{V \in C_{N_{1}}: V \cap E \neq \varnothing\right\}$. By property (e) of $\S 1$, with $\left|Z_{1}\right|$ as the cardinality of $Z_{1}$,

$$
a_{N_{1}} \cdot\left|Z_{1}\right|=\lambda\left(\bigcup Z_{1}\right) \geq \lambda(E)>(1-\varepsilon) a_{N_{0}}
$$

Thus,

$$
1-b_{N_{0}} a_{N_{1}}\left|Z_{1}\right|<\varepsilon
$$

Since $s_{N_{1}}(S)$ is constant on elements of $C_{N_{1}}$,

$$
\left|s_{N_{1}}(S, x)\right|<\varepsilon_{1} \quad \text { for } x \in \bigcup Z_{1} \text {. }
$$

Now pick $U_{1} \in C_{N_{1}}$ such that

$$
\left|\left(S, \xi_{U_{1}}\right)\right|=\max \left\{\left|\left(S, \xi_{V}\right)\right|: V \subseteq U_{0}, V \in C_{N_{1}} \backslash Z_{1}\right\} .
$$

If $\bigcup Z_{1}=U_{0}$, pick any $U_{1} \in C_{N_{1}}$ with $U_{1} \subseteq U_{0}$.

Since $s_{n}(S, x) \rightarrow 0 \lambda$-a.e. on $U_{1}$, induction and the above argument shows that there are sequences $\left(\varepsilon_{j}\right)_{j=1}, N_{j}>N_{j-1}, Z_{j} \subseteq C_{N_{j}}$, and $U_{j} \in C_{N_{j}}$ such that

$$
\begin{gathered}
\varepsilon_{j}=\varepsilon /\left(2^{j} a_{N_{j-1}} \prod_{p=1}^{j-1}\left(b_{N_{p}} a_{N_{p-1}}-\left|Z_{p}\right|\right)\right) \\
1-b_{N_{j-1}} a_{N_{j}}\left|Z_{j}\right|<\varepsilon \\
\left|s_{N_{j}}(S, x)\right|<\varepsilon_{j} \quad \text { for } x \in \bigcup Z_{j}, \text { and } \\
\left|\left(S, \xi_{U_{j}}\right)\right|=\max \left\{\left|\left(S, \xi_{V}\right)\right|: V \subseteq U_{j-1}, V \in C_{\left.N_{j} \backslash Z_{j}\right\}}\right.
\end{gathered}
$$

unless $\bigcup Z_{j}=U_{j-1}$, in which case $U_{j}$ is any element of $C_{N_{j}}$ contained in $U_{j-1}$.

Now,

$$
\begin{aligned}
\left|\left(S, \xi_{U}\right)\right| & \leq \sum\left\{\left|\left(S, \xi_{V}\right)\right|: V \in Z_{1}\right\}+\sum\left\{\left|\left(S, \xi_{V}\right)\right|: V \subseteq U_{0}, V \in C_{N_{1}} \backslash Z_{1}\right\} \\
& \leq a_{N_{1}} \varepsilon_{1}\left|Z_{1}\right|+\left(b_{N_{1}} a_{N_{0}}-\left|Z_{1}\right|\right)\left|\left(S, \xi_{U_{1}}\right)\right|
\end{aligned}
$$

since $\left(S, \xi_{V}\right)=a_{N_{1}} s_{N_{1}}(S, x)$ for $x \in V \in C_{N_{1}}$ and $\left|\left\{V: V \in C_{N_{1}}, V \subseteq U\right\}\right|=$ $b_{N_{1}} a_{N_{0}}$. But $a_{N_{1}}\left|Z_{1}\right| \leq a_{N_{0}}$, so

$$
\left|\left(S, \xi_{U}\right)\right| \leq a_{N_{0}} \varepsilon_{1}+\left(b_{N_{1}} a_{N_{0}}-\left|Z_{1}\right|\right)\left|\left(S, \xi_{U_{1}}\right)\right| .
$$


(9)

Continuing in this manner and recalling (4), we get

$$
\begin{aligned}
\left|\left(S, \xi_{U}\right)\right| \leq & \varepsilon_{1} a_{N_{0}}+\varepsilon_{2} a_{N_{1}}\left(b_{N_{1}} a_{N_{0}}-\left|Z_{1}\right|\right)+\cdots \\
& +\varepsilon_{j} a_{N_{j-1}} \prod_{k=1}^{j-1}\left(b_{N_{k}} a_{N_{k-1}}-\left|Z_{k}\right|\right)+\left|\left(S, \xi_{U_{j}}\right)\right| \prod_{k=1}^{j}\left(b_{N_{k}} a_{N_{k-1}}-\left|Z_{k}\right|\right) \\
= & \varepsilon\left(1-2^{-j}\right)+\left|\left(S, \xi_{U_{j}}\right)\right| \prod_{k=1}^{j}\left(b_{N_{k}} a_{N_{k-1}}-\left|Z_{k}\right|\right) .
\end{aligned}
$$

Choose $x_{0} \in \bigcap_{j=1}^{\infty} U_{j}$ by compactness. Then $\left(S, \xi_{U_{j}}\right)=a_{N_{j}} s_{N_{j}}\left(S, x_{0}\right)$ by definition, so

$$
\begin{aligned}
\left|\left(S, \xi_{U}\right)\right| & \leq \varepsilon\left(1-2^{-j}\right)+\left|s_{N_{j}}\left(S, x_{0}\right)\right| a_{N_{j}} \prod_{k=1}^{j}\left(b_{N_{k}} a_{N_{k-1}}-\left|Z_{k}\right|\right) \\
& =\varepsilon\left(1-2^{-j}\right)+\left|s_{N_{j}}\left(S, x_{0}\right)\right| a_{N_{0}} \prod_{k=1}^{j}\left(1-a_{N_{k}} b_{N_{k-1}}\left|Z_{k}\right|\right) \\
& \leq \varepsilon\left(1-2^{-j}\right)+M a_{N_{0}} \varepsilon_{j},
\end{aligned}
$$

where $M=\sup \left\{\left|s_{n}\left(S, x_{0}\right)\right|: n \geq 0\right\}$. Let $j \rightarrow \infty$ to get $\left|\left(S, \xi_{U}\right)\right| \leq \varepsilon$. Let $\varepsilon \rightarrow 0$ to get $\left(S, \xi_{U}\right)=0$, as desired.

This theorem is the key step in our proof of an analog of Bary's theorem on the union of closed $U$-sets. Before we state and prove this result, we need some terminology and some lemmas.

Up to now, we have ignored the multiplicative structure of $\operatorname{TP}(X)$. Quite obviously, it is closed under pointwise multiplication. This fact allows us to define a module structure on $\mathrm{QM}(X)$ as follows.

Definition. Let $f \in \mathrm{TP}(X)$ and $S \in \mathrm{QM}(X)$. Define $f S \in \mathrm{QM}(X)$ by

$$
(f S, g)=(S, f g) \quad \text { for } g \in \operatorname{TP}(X) \text {. }
$$

This evidently defines a linear functional on $\operatorname{TP}(X)$ and so an element of $\mathrm{QM}(X)$. Our next lemma is a simple form of a Rajchman result.

LEMMA 4.4. Let $S \in \mathrm{QM}(X)$ and $U \in C_{n}$. Then for $m \geq n, s_{m}\left(\xi_{U} S, x\right)=$ $\xi_{U}(x) s_{m}(S, x)$ for all $x \in X$.

PROOF.

$$
\begin{aligned}
s_{m}\left(\xi_{U} S, x\right) & =\left(1 / a_{m}\right)\left(\xi_{U} S, \xi_{U(m, x)}\right)\left(=\left(S, \xi_{U} \xi_{U(m, x)}\right)\left(1 / a_{m}\right)\right. \\
& = \begin{cases}\left(1 / a_{m}\right)\left(S, \xi_{U(m, x)}\right) & \text { if } x \in U, \\
0 & \text { if } x \notin U,\end{cases} \\
& =\xi_{U}(x) s_{m}(S, x) .
\end{aligned}
$$

The third equality follows since $m \geq n$ and $x \in U$ implies $U(m, x) \subseteq U$ while $m \geq n$ and $x \notin U$ implies $U(m, x) \cap U=\bar{\varnothing}$.

DEFinition. Let $A \subseteq \mathrm{QM}(X)$. A subset $E$ of $X$ is called a set of uniqueness for $A$ (a $U$-set for $A$, or simply a $U$-set) if $S \in A$ and $s_{n}(S, x) \rightarrow 0$ for all $x \notin E$ implies $S=0$. 
Thus Corollary 3.5 says that the empty set is a $U$-set for $\mathrm{QM}(X)$, while Theorem 4.3 says that every $\lambda$-null set is a $U$-set for the collection of quasi-measures $S$ such that $\varlimsup_{n \rightarrow \infty}\left|s_{n}(S, x)\right|<\infty$ for all $x \in X$.

If $A$ is a linear subspace of $\mathrm{QM}(X)$, we say that $A$ satisfies condition $(L)$ if

(a) every $\lambda$-measurable $U$-set for $A$ is $\lambda$-null, and

(b) $S \in A$ and $U \in \bigcup_{n=0}^{\infty} C_{n}$ implies $\xi_{U} S \in A$.

See $[\mathbf{1 1}]$ for an investigation of the Walsh series case.

We close this section with our analog of Bary's theorem. See [7] for the classical case and $[\mathbf{1 1}, \mathbf{1 2}]$ for the Walsh series case. First we need a lemma.

LEMMA 4.5. Let $A \subseteq \mathrm{QM}(X)$ satisfy condition $(L)$. Let $E$ be a closed $U$-set for $A$ with $E \subseteq U \in C_{m}$. If $S \in A$ with

(i) $\varlimsup_{n \rightarrow \infty}\left|s_{n}(S, x)\right|<\infty$ for $x \in U \backslash E$, and

(ii) $\lim _{n \rightarrow \infty} s_{n}(S, x)=0 \lambda$-a.e. in $U$, then $\lim _{n \rightarrow \infty} s_{n}(S, x)=0$ everywhere in $U$.

Proof. Let $x \in V \in C_{j}$ with $V \subseteq U$ and $V \cap E=\varnothing$. For $n \geq j$, Lemma 4.4 gives

$$
s_{n}\left(\xi_{V} S, y\right)=\xi_{V}(y) s_{n}(S, y) \quad \text { for all } y \in X
$$

Thus, by (i),

$$
\varlimsup_{n \rightarrow \infty}\left|s_{n}\left(\xi_{V} S, y\right)\right|<\infty \quad \text { for all } y \in X
$$

By (ii),

$$
\lim _{n \rightarrow \infty} s_{n}\left(\xi_{V} S, y\right)=0 \quad \lambda \text {-a.e. in } X
$$

By Theorem 4.3, $\xi_{V} S=0$. Thus, for our fixed $x$,

$$
s_{n}(S, x)=\xi_{V}(x) s_{n}(S, x)=s_{n}\left(\xi_{V} S, x\right)=0 \quad \text { for } n \geq j .
$$

Since $E$ is closed,

$$
\lim _{n \rightarrow \infty} s_{n}(S, x)=0 \quad \text { for all } x \in U \backslash E .
$$

Applying Lemma 4.4 again, we see that

$$
\lim _{n \rightarrow \infty} s_{n}\left(\xi_{U} S, x\right)=0 \quad \text { for all } x \in X \backslash E .
$$

Since $\xi_{U} S \in A$ and $E$ is a $U$-set for $A, \xi_{U} S=0$. The lemma follows.

Now, our version of Bary's theorem.

THEOREM 4.6. Let $A \subseteq \mathrm{QM}(X)$ satisfy condition $(L)$. Then the countable union of closed $U$-sets for $A$ is again a $U$-set for $A$.

Proof. Call $U$-sets for $A$ simply $U$-sets.

Let $\left\{E_{n}\right\}_{n=0}^{\infty}$ be closed $U$-sets, and set $E=\bigcup_{n=0}^{\infty} E_{n}$. By part (a) of condition $(L), \lambda(E)=0$. Assume $S \in A \backslash\{0\}$ with $\lim _{n \rightarrow \infty} s_{n}(S, x)=0$ for $x \notin E$. We will obtain a contradiction.

Set $N=\left\{x \in X: s_{n}(S, x)\right.$ is unbounded $\}$. By Theorem 4.3, $N \neq \varnothing$, and $N=\bigcap_{k=1}^{\infty} \bigcup_{n=1}^{\infty}\left\{x \in X:\left|s_{n}(S, x)\right|>k\right\}$ is a $G_{\delta}$ in the compact space $X$.

Since $s_{n}(S, x) \rightarrow 0$ for $x \notin E, N \subseteq E$. Thus $N=\bigcup_{n=0}^{\infty}\left(N \cap E_{n}\right)$. Each $N \cap E_{n}$ is closed in $N$, so the Baire category theorem gives an open set $U$ such that $\varnothing \neq U \cap N \subseteq N \cap E_{n_{0}}$ for some $n_{0}$, i.e. some $E_{n_{0}}$ is somewhere dense in $N$. We may take $U$ to be in the base $\bigcup_{n=0}^{\infty} C_{n}$ for the topology of $X$. 
Now, $U \cap N \subseteq N \cap E_{n_{0}} \subseteq E_{n_{0}} \cap U$. But $E_{n_{0}} \cap U$ is a $U$-set, being a subset of the $U$-set $E_{n_{0}}$. It is also closed since both $E_{n_{0}}$ and $U$ are. But

$$
\lim _{n \rightarrow \infty} s_{n}(S, x)=0 \quad \lambda \text {-a.e. in } U,
$$

and

$$
\varlimsup_{n \rightarrow \infty}\left|s_{n}(S, x)\right|<\infty \quad \text { for } x \in U \backslash N \subseteq U \backslash\left(E_{n_{0}} \cap U\right) .
$$

By Lemma $4.5, \lim _{n \rightarrow \infty} s_{n}(S, x)=0$ everywhere in $U$. But this contradicts the fact that $U \cap N \neq \varnothing$.

COROLlary 4.7. If $A \subseteq \mathrm{QM}(X)$ satisfies condition $(L)$ and if singletons are $U$-sets for $A$, then any countable set is a $U$-set for $A$.

\section{REFERENCES}

1. W. R. Wade and K. Yoneda, Uniqueness and quasi-measures on the group of integers of a p-series field, Proc. Amer. Math. Soc. 84 (1982), 202-206.

2. K. Yoneda, On generalized uniqueness theorems for Walsh series, Acta Math. Hungar. 43 (1984), 209-217.

3. N. J. Fine, On Walsh functions, Trans. Amer. Math. Soc. 65 (1949), 373-414.

4. E. Hewitt and K. Stromberg, Real and abstract analysis, Springer-Verlag, New York, 1965.

5. E. Hewitt and K. A. Ross, Abstract harmonic analysis. II, Springer-Verlag, New York, 1970.

6. N. J. Fine, Fourier-Stieltjes series of Walsh functions, Trans. Amer. Math. Soc. 86 (1957), 246255.

7. A. Zygmund, Trigonometric series, Cambridge Univ. Press, Cambridge, 1979.

8. R. B. Crittenden and V. L. Shapiro, Sets of uniqueness on the group $2^{\omega}$, Ann. of Math. 81 (1965), 550-564.

9. W. Wade, A uniqueness theorem for Haar and Walsh series, Trans. Amer. Math. Soc. 141 (1969), 187-194.

10. __ Growth conditions and uniqueness for Walsh series, Michigan Math. J. 24 (1977), 153-155.

11. K. Yoneda, Summing generalized closed U-sets for Walsh series, Proc. Amer. Math. Soc. 94 (1985), 110-114.

12. W. Wade, Summing closed U-sets for Walsh series, Proc. Amer. Math. Soc. 29 (1971), 123-125.

Department of Mathematics, Kansas State University, Manhattan, Kansas 66506

Current address: Department of Mathematical Sciences, Northern Illinois University, DeKalb, Illinois 60115-2888 